\title{
Some Observational Limitations of Extinction Measurements in the Rocket Ultraviolet
}

\author{
JAN BORGMAN \\ Kapteyn Observatory \\ Roden, The Netherlands
}

TVEN BEFore any MEASUREMENT of stellar fluxes in the ultraviolet E had been obtained, predictions were being made on the basis of stellar atmosphere models. At the moment, it seems that such com. putations (for example, see ref. 1) are in reasonable agreement with actual observations from rockets (ref. 2) in the spectral region down to about $2000 \AA$. At shorter wavelengths it is necessary to take into account the appreciable blanketing by spectral lines which unavoidably is present in the transmission bands of any wide-band photometric system, even in the case of early B stars, as has been shown in reference 3 .

It must be anticipated that the reduction of extinction measurements in the UV from spaceborne instruments will follow the same procedure that has been used so far: stars with the same intrinsic energy distribution but with different amounts of reddening material in the line of sight are compared, the difference in energy distribution being attributed to the wavelength dependence of interstellar extinction.

In past investigations of interstellar extinction in the visible and near UV, assumptions about equal energy distribution have sometimes been challenged, even if the stars concerned had the same MK classification and their spectra at moderate dispersion showed no complicated structure or differences of any photometric significance. This situation would undoubtedly become more critical in the UV, particularly below $1500 \AA$ where the spectra of $O$ and B stars must be expected to be quite complicated. Hence, when deriving extinction data from wide-band measurements below $1500 \AA$, one faces a problem which looks somewhat like the equivalent situation in the infrared when extinction measurements have to be derived from M-type supergiants. For the moment, it is assumed that wide-band photometry, aimed at obtaining extinction data, should be restricted to the wavelength region $\lambda>1500 \AA$. 
It is likely that significant measurements of the reddening law in the UV will be made from stabilized satellites rather than from rockets, as the stars to be investigated are faint and are to be found in densely populated regions of the sky. Hence the desirability is evident of predicting the encountered fluxes in order to compile a sensible and realistic observing program, which can be carried out by the experiment in terms of its dynamic range and available observing time.

To this end fluxes have been computed of reddened stars by using the relation

where

$$
F\left(\lambda, V_{o} \quad A_{v}\right)=F(\lambda, 0,0) \times 10^{-0.4\left(V_{0}+c_{A} A_{v}\right)}
$$

$F(\lambda, 0,0)$ is the intrinsic flux in photons $/ \mathrm{cm}^{2} \cdot \mathrm{A}-\mathrm{sec}$ at wavelength $\lambda$ $V_{o}$ is the apparent visual magnitude if the absorption has been removed

$A_{V} \quad$ is the visual absorption

$c_{\lambda} \quad$ represents the extinction law, $c_{\lambda}=A_{\lambda} / A_{V}$

Typical flux values at four wavelengths for a B0 star of apparent visual magnitude $V=10$ and visual absorption $A_{V}=2$ (hence $V_{0}=8$ ) are given in table I. The adopted values of $c_{\lambda}$ are also tabulated; they

TABLE I. - Adopted Extinction Law and Flux Values for a B0 Star $\left[V=10, A_{V}=2, V_{o}=8\right]$

\begin{tabular}{|c|c|c|}
\hline$\lambda, \AA$ & $c_{\lambda}$ & $F$, photons $/ \mathrm{cm}^{2} \AA \AA \AA-s e c$ \\
\hline & & \\
3300 & 1.70 & 0.09 \\
2600 & 2.07 & .07 \\
2200 & 2.58 & .04 \\
1500 & 2.58 & .08 \\
\hline
\end{tabular}

are based on the data given in reference 4 , except for the 1500 - $\AA$ extinction which has been assumed to be the same as that at $2200 \AA$.

Suppose the star of table $I$ is observed with an 8-inch telescope in $200-\AA$ bands centered at the four wavelengths in the table, with an efficiency of 4 percent (resulting from an optical efficiency of 20 percent and a detector efficiency of 20 percent). It is found that the star would develop 100 primary photoelectrons in the 200 - $\AA$ bandpass at $2200 \AA$ in 1 second and correspondingly more in the other three wavelength regions. The counting of 10000 events would take 100 seconds (100 percent collection efficiency and no dark current assumed). This reasonable time being assumed, stars are selected for an observing program, intended for a study of the extinction law. It is likely that one would want to observe stars which have already been studied in other wavelength regions from the ground. A sensible target could be the observa- 
tion of the 1259 stars in the catalogue of reference 5 . In that catalogue, 1193 stars have spectral types earlier than $\mathrm{Al}$; for these stars, fluxes have been computed following the procedure described earlier. In addition, 975 stars appear to have fluxes at all four wavelengths in excess of $10^{-2}$ photons $/ \mathrm{cm}^{2}-\AA-\mathrm{sec}$ and are considered to be observable in terms of photon statistics. A breakdown of the figures is given in table II.

TABLE II. - Numbers of Several Star Spectral Types a

\begin{tabular}{|c|c|c|c|}
\hline Spectral type & Catalogue & Flux computed & Flux $>10^{-2}$ photons $/ \mathrm{cm}^{2} \cdot \AA$-sec \\
\hline O & & 200 & 164 \\
BO-B1.5 & & 637 & 533 \\
B2-A0 & & 356 & 278 \\
\hline Total & 1259 & 1193 & ' 975 \\
\hline
\end{tabular}

a Data obtained from reference 5.

${ }^{b}$ Of these 975 stars, $A_{V}<1$ magnitude for $94 ; 1 \leqslant A_{V}<2$ for 485; and $A_{V} \geqslant 2$ for 396 .

The actual extinction information relative to extinction measurements in the visible and infrared must come from stars with a visual extinction of at least 1 magnitude, of which there are 881. If the one-third of the Milky Way not represented in Hiltner's catalogue (ref. 5) and the additional observation of some unreddened stars is accounted for, the total number of stars would be about 1500 , the observing time ranging from a few seconds to approximately 7 minutes on the basis of the adopted instrumental specifications.

In the case of Orbiting Astronomical Observatory Al (OAO-Al), not all these stars could be observed because the guidance system is not compatible with the small diaphragm required to isolate many of the stars in galactic clusters. However, outside of clusters, much information on the extinction law should be accessible to a moderately sized instrument with a field of view of a few minutes of arc.

\section{REFERENCES}

1. Underhilu, A. B.: A Program for Computing Early-Type Model Atmospheres and Testing the Flux Integral. Pub. Dominion Astrophys. Obs., vol. 11, 1962, p. 433.

2. Strömgren, B.: Comparison of Observed and Theoretically Calculated Intensities in the Continuous Spectra of Main-Sequence B Stars. Rev. Mod. Phys., vol. 36, 1964, p. 532 .

3. Mihalas, D. M.; and Morton, D. C.: A Model for a BIV Star with Line Blanketing. Astrophys. J., vol. 142, 1965, p. 253.

4. Boggess, A.; and Borgman, J.: Interstellar Extinction in the Middle Ultraviolet. Astrophys. J., vol. 140, 1964, p. 1636.

5. Hiltner, W. A.: Photoelectric, Polarization and Spectrographic Observations of $O$ and B Stars. Astrophys. J. Suppl. Ser., vol. 2, 1956, p. 389. 


\section{DISCUSSION}

Nandy: When we compare two stars to derive the extinction curve, we assume that their intrinsic energy distribution is the same for reddened and unreddened stars. The spectral behavior of the stars is often determined from spectrophotometric measurements in the visual wavelength region. Can we assume that stars which have the same spectral colors based on measurements in blue and visual wavelengths will have the same energy distribution in the far ultraviolet? Is the influence of metal abundances likely to be more important in the ultraviolet?

Borgman: Down to $2000 \AA$, for the stars that have been investigated, a reasonable agreement has been found between atmospheric radiation fields computed from stellar atmosphere models and the observations. Below $2000 \AA$ I think the procedure is much more questionable. But any questions of this kind can be avoided by observing a sufficiently large number of stars and seeing how the scatter is around the reddening path in a color-color diagram. If it is small, then one feels reasonably certain that the simplest interpretation is valid.

Nandy: That is why it is dangerous to derive any conclusion from measurements of only a few stars in the ultraviolet. We have to observe many more stars.

Borgman: Yes, certainly anyone would agree with you; but having not so many data available, I do not consider it unreasonable to analyze the few stars that have been observed. In the case of Boggess' results we made these color-color plots and we were quite pleased that the scatter wasn't much larger than we had hoped for. We didn't publish the color-color plots, but anyone could make them up from the numbers that we published and see what they are worth. I agree with you that more observations are desirable; but many of these observations have to be made from satellites in order to increase the accuracy. These highly reddened objects are faint and require long integration times.

Wickramasinghe: Do you think the spectrophotometric method that Stecher and Milligan are using is any improvement on the use of filters in the ultraviolet?

Borgman: No, I am afraid that I often prefer reasonably wide-band filter measurements just because I think that the final analysis of any scanner observations is always such that one simply selects points from the scans. Therefore, rather than scanning through each point I prefer integrating the information.

Wickramasinghe: But the scanner method would reproduce narrow absorption peaks which you couldn't detect with a filter.

Borgman: And you think of absorption peaks in terms of interstellar absorption? 
Comment: Yes, I think so.

Borgman: I see; well, I don't believe that they can be seen on the scans that have been obtained so far.

Spitzer: You mentioned the complications of blanketing in the wide-band measures down to $1500 \AA$. We now have preliminary sounding rocket data at Princeton on the absorption lines in two Scorpius stars ( $\pi$ Sco and $\delta$ Sco). The spectrograms have a resolution of about $1 \AA$, but they are not really suitable for spectrophotometry. They do suggest that down to $1500 \AA$ the correction for line absorption due to blanketing may be quite small. Except for the two Si IV lines, and the corresponding $\mathrm{C} I V$ doublet, the other lines are relatively weak.

Borgman: I see; this is something which is rather promising because I thought that one should refrain from going beyond $1500 \AA$ because of what Morton had said earlier, and in a sense it confirms what Byram and Chubb have found.

Hall: What would be the intensity of the galactic backgreund of these wavelengths? Do you have any idea-compared with the stars?

Borgman: No one knows. This is, of course, a problem, and that is why I said "some" observational limitations in the title. A bright sky might mean that in an experiment like the OAO-Al the diaphragm sizes are too large to measure the stars in Hiltner's catalogue.

Hall: Isn't the scattering by the optics at these very short wavelengths another problem?

Borgman: That can be reasonably investigated and cured in the lab. I think that the experimenters are confident that this won't be a limitation.

Hall: I didn't quite understand why there was so much flux at $1500 \AA$.

Borgman: That is just because I took the same extinction there as at $2200 \AA$, and the star simply gets brighter.

Hall: You mean it is brighter at $\lambda=3300 \AA$, dips down, and then comes back again?

Borgman: Yes, but that is simply a disguise, of course. It is the interstellar reddening that makes it fainter down to $\lambda=2200 \AA$ and because I did choose not to increase the interstellar reddening beyond $\lambda=2200 \AA$, the star itself wins again and finally doubles the output.

Strömgren: As material grows in the coming years this analysis of the color-color diagram, I think, is going to be very important. Dr. Spitzer pointed out that if we don't go much beyond $\lambda=2000 \AA$ the additional parameter (blanketing) should not be a serious one. It probably is well to look out for parameters that might cause scatter. The rotation parameter is important here. It is hoped that the model atmosphere work will be extended into the far UV as more data on the extinction in this region become available. It is a difficult extension to account for the rotation parameter as well. 
Nandy: Work on rotating stars that has been done in Edinburgh by Guthrie shows that there is a difference in the intrinsic colors depending on whether the star is observed pole-on or equator-on.

Stecher: In reference to the application by Dr. Strömgren about gravity darkening, Collins has found more than one magnitude of difference for a very rapidly rotating star. If the additional Morgan classification for the early $B$ stars is used, there is quite a spread in temperature between, for example, a $\mathrm{B} 2$ star and a $\mathrm{B} 0$ star. The temperature range is quite large and it may be more accurate to use classifications such as Dr. Strömgren's filters to determine whether or not the two stars are similar. This is always going to be a problem - are they really similar especially when the excitation spectrum doesn't distinguish absolutely.

Strömgren: I think it is well to emphasize that, because as we go to this wide wavelength band we will probably need the results of quite extensive model atmosphere calculations in order to interpret these scatter diagrams. If you look in the wavelength regions that can be measured from the ground and then limit yourself to main sequence stars, you can subdivide according to beta index, but the evidence is that this does not affect the continuous curve much, even in the ultraviolet. The rotational effects imply two parameters: the angle and the speed of rotation, and I think it will become necessary, in order to analyze the data over a wide wavelength range, to go beyond what we can observe in the visual region for our calculations. However, this will be necessary only when we aim at quite a high standard of accuracy; I wouldn't say that it is very important at the present time.

Borgman: Whatever theoretical work will develop, data can be obtained almost without any theory on the stellar radiation field at all, simply by observing color indices and plotting them to see how the scatter looks. This sounds like a crude approach; but, on the other hand, there is little to hold against it. I would also like to add to what Dr. Stecher said a moment ago about the accuracy that one really needs in the temperature, that it is gratifying to remember that the temperature reddening looks a little bit like interstellar reddening. If it were the same you wouldn't notice it at all.

Greenberg: I must comment on temperature reddening looking like interstellar reddening. Over a limited spectral range it looks like $\lambda^{-1}$. I can't remember how far into the UV you can carry it and still say that the temperature reddening might look like the interstellar reddening.

Wickramasinghe: Certainly this would apply in the visible region, but I think the differences would be rather large in the ultraviolet.

Greenberg: It is my recollection that it would not apply in the ultraviolet.

Borgman: We shouldn't be too exacting about such a statement as I made. I think that the observations are still so crude that it holds. 